\title{
LOCALLY CR SPHERICAL THREE MANIFOLDS
}

\author{
HOWARD JACOBOWITZ
}

\begin{abstract}
Every open and orientable three manifold has a CR structure which is locally equivalent to the standard CR structure on $S^{3}$.
\end{abstract}

The standard CR structure on $S^{3}$ is the one induced by the usual complex structure on $R^{4}$. A CR structure on a three dimensional manifold is said to be locally spherical if for each point there exist an open neighborhood $U$ and a CR diffeomorphism of $U$ onto an open subset of $S^{3}$. There are many results about compact locally spherical three manifolds (for instance, [1, 2], 4], [5, 6]) which show that these manifolds are special. The situation is quite different for open manifolds. This easy observation seems not to be in the literature. (There are some interesting constructions on special open manifolds, particularly in connection with complex hyperbolic geometry [3, 8.)

Theorem. Every open and orientable three dimensional manifold admits a locally spherical CR structure.

Remark 1. The CR structure may be taken to be $C^{\omega}$.

If we remove one point from $S^{3}$ and choose any diffeomorphism of this new manifold to $R^{3}$, we end up with a CR structure on $R^{3}$. (Usually this diffeomorphism is taken so that we end up with the $\mathrm{CR}$ structure induced on the hyperquadric $\left\{(z, w) \in C^{2}|\Im w=| z^{2} \mid\right\}$.) Any map

$$
F: M \rightarrow R^{3}
$$

which is a local diffeomorphism may be used to pull this CR structure on $R^{3}$ back to $M$. The result is a locally spherical CR structure on $M$. The existence of some $F$ is a well-known topological fact, first proved by Whitehead 9. See also Phillips [7], Corollary 8.2 and the references cited there.

Remark 2. The conclusion of the theorem also holds for any parallelizable manifold of odd dimension.

\section{REFERENCES}

[1] Burns, D. M., Jr.; Epstein, C. L. A global invariant for three-dimensional CR-manifolds. Invent. Math. 92 (1988), no. 2, 333348.

[2] Chng, Jih Hsin; Lee, John M. The Burns-Epstein invariant and deformation of CR structures. Duke Math. J. 60 (1990), no. 1, 221254.

[3] Falbel, Elisha A spherical CR structure on the complement of the figure eight knot with discrete holonomy. J. Differential Geom. 79 (2008), no. 1, 69110.

[4] Falbel, Elisha; Gusevskii, Nikolay Spherical CR-manifolds of dimension 3. Bol. Soc. Brasil. Mat. (N.S.) 25 (1994), no. 1, 3156.

Date: March 21, 2013

Key words and phrases. CR, locally spherical.

2010 Mathematics Subject Classification: 32V05. 
[5] Isaev, Alexander Spherical tube hypersurfaces. Lecture Notes in Mathematics, 2020. Springer, Heidelberg, 2011.

[6] Le, A., Cartan connections for CR manifolds. Manuscripta Math. 122 (2007), no. 2, 245264.

[7] Phillips A., Submersions of open manifolds, Topology 6(1967), 171-206.

[8] Schwartz, R. E., Spherical CR geometry and Dehn surgery. Annals of Mathematics Studies, 165. Princeton University Press, Princeton, NJ, 2007.

[9] Whitehead J. H. C., The Immersion of an Open 3-Manifold in Euclidean 3-Space, Proc. London Math. Soc. (3), 11(1961),81-90.

Department of Mathematical Sciences, Rutgers University, Camden, NJ 08102

E-mail address: jacobowi@camden.rutgers.edu 